\title{
Electrically Controlled Adsorption of Oxygen in Bilayer Graphene Devices
}

\author{
Yoshiaki Sato, ${ }^{1, *}$ Kazuyuki Takai, ${ }^{1}$ and Toshiaki Enoki ${ }^{1}$ \\ ${ }^{1}$ Department of Chemistry, Tokyo Institute of Technology, 2-12-1 Ookayama, \\ Meguro-ku, Tokyo, 152-8551, Japan
}

\begin{abstract}
We investigate the chemisorptions of oxygen molecules on bilayer graphene (BLG) and its electrically modified charge-doping effect using conductivity measurement of the field effect transistor channeled with BLG. We demonstrate that the change of the Fermi level by manipulating the gate electric field significantly affects not only the rate of molecular adsorption but also the carrierscattering strength of adsorbed molecules. Exploration of the charge transfer kinetics reveals the electrochemical nature of the oxygen adsorption on BLG. [This document is the unedited Author's version of a Submitted Work that was subsequently accepted for publication in Nano Letters, (C)American Chemical Society after peer review. To access the final edited and published work see http://dx.doi.org/10.1021/nl202002p.]
\end{abstract}

Keywords: Graphene; charge transfer; field effect transistor; electron transport; mobility; band gap

It has been a central topic of surface science how to control the adsorption and desorption in order to to bring out desirable features and functionalities by adsorbed molecules. Tuning the electronic features of solid surfaces has an important implication in that molecular chemisorptions and catalytic reactions are determined by them ${ }^{1,2}$. In particular for graphene, the twodimensional honeycomb carbon lattice, in which the conduction $\pi^{*}$-band and the valence $\pi$-band contact to each other at the "Dirac point" giving a feature of zero-gap semiconductor ${ }^{3}$, the control of chemisorption is a critical issue since chemisorption directly leads to altering every electronic property of graphene. Other than the electron/hole doping ${ }^{4}$ owing to the charge transfer between graphene and the adsorbed molecules, widely known are the charged impurity effect on the electron transport ${ }^{5-7}$, lattice deformation ${ }^{8}$, and opening the band gap due to asymmetric adsorption ${ }^{9-11}$. Aside from the macroscopic spatially-controlled adsorption that is achieved using nano-device fabrication technique ${ }^{12}$, microscopic control of adsorption structure is of great importance because the aforementioned adsorption effects are altered by the local structure of adsorbate, e.g., whether the adsorbed molecules are arranged in a random or superlattice structure ${ }^{10,13}$, or whether the molecules are adsorbed individually ${ }^{4,14}$ or collectively (in dimers ${ }^{15}$ or clusters $\left.^{16,17}\right)$. For the first step to realize such an advanced control of adsorption, the methods to utilize the interaction between the adsorbed molecules and graphene for it are to be explored.

The principal impetus in the present study is to control the charge transfer between graphene and the adsorbed molecules by tuning the Fermi level of graphene, which is readily accomplished in the field effect transistor (FET) structure. When $\mathrm{SiO}_{2} / \mathrm{Si}$ substrate is used as the back-gate insulator of the FET, the tuning range of the Fermi level of graphene by the application of the gate voltage is at the extent of several $\pm 0.1 \mathrm{eV}^{18}$ which would be sufficient to alter the chemical reactivity on the surface. Besides, the additional charge and the gradient of electric potential generated by the gate electric
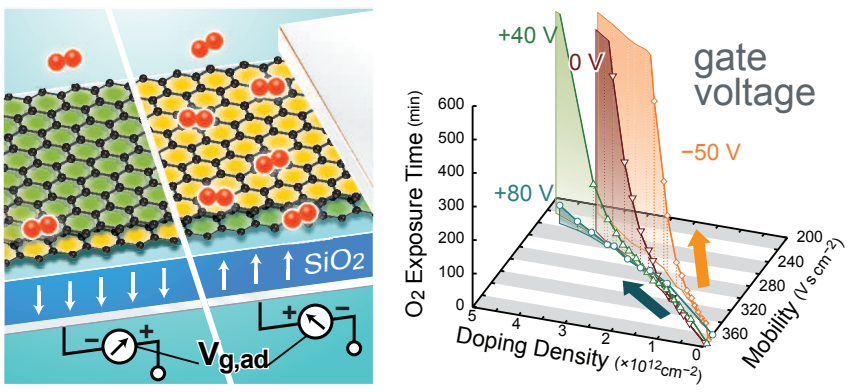

field are expected to change the polarization of adsorbed molecules ${ }^{19,20}$ and to modify the charge distribution on graphene layers and the adsorbed molecules ${ }^{21-25}$ leading to, e.g., the change in the ease of migration of molecules adsorbed on graphene ${ }^{26}$. Some research ${ }^{27-29}$ argues that the change in the Fermi level caused by a gate electric field activates electrochemical redox reactions and the accompanying charge transfer causes hysteresis of the source-drain current in graphene FET, yet there has been no investigation that elucidates the relation between the kinetics of adsorption to graphene and gate electric field. In this study we investigated gate-tuned molecular oxygen adsorption through systematic measurements of conductivity using mainly bilayer graphene (BLG).

The back-gated BLG-FETs were fabricated on $\mathrm{SiO}_{2}$ (300 $\mathrm{nm}$ thick) on heavily n-doped silicon substrate by means of photolithography. The channel length and width were $6 \mu \mathrm{m}$ and $3.5 \mu \mathrm{m}$, respectively. Prior to measurement, we repeated vacuum annealing $\left(210^{\circ} \mathrm{C}, 10 \mathrm{~h}\right)$ to remove the adsorbed moisture and contaminants on the surface until no more changes in the gate-dependent conductivity $\sigma$ were eventually seen. After the annealing, the BLG-FET exhibited its pristine nature, that is, ambipolar transport properties with a conductivity minimum around $V_{\mathrm{g}}=V_{\mathrm{CNP}}^{0}<8 \mathrm{~V}$ giving the "charge neutrality point (CNP)" with electrons and holes in BLG being equal in density. The Drude mobility $\mu(n)$ was estimated to be $\sim 1 \times 10^{3} \mathrm{~cm}^{2} \mathrm{~V}^{-1} \mathrm{~s}^{-1}$ from the equation $\mu(n)=\sigma / e|n|$, where $n$ is the carrier density with $n=\left(c_{\mathrm{g}} / e\right)\left(V_{\mathrm{g}}-V_{\mathrm{CNP}}\right)\left(c_{\mathrm{g}} / e=7 \times 10^{10} \mathrm{~cm}^{-2} \mathrm{~V}^{-1}\right.$; 
a

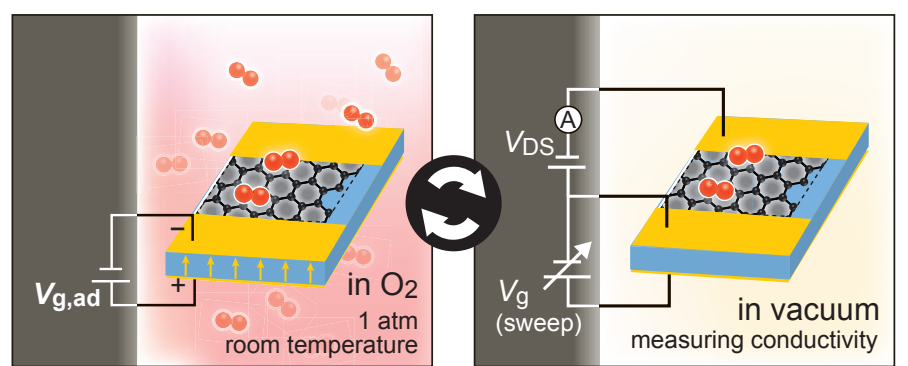

b

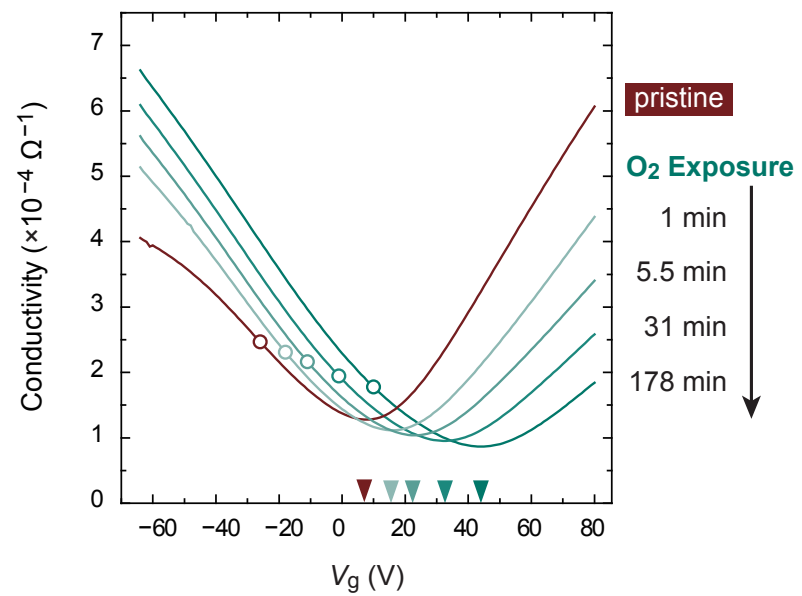

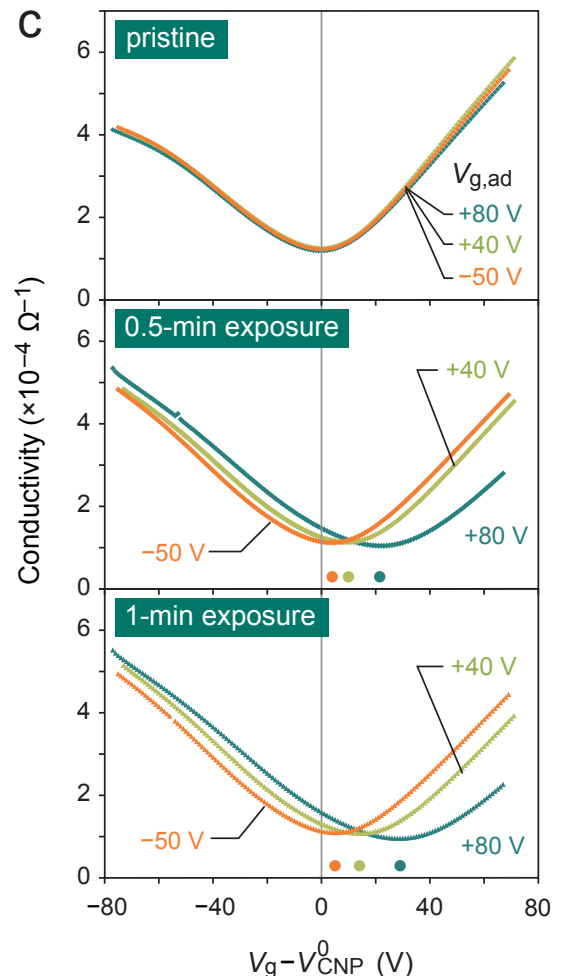

FIG. 1: (a) Schematic of the measurement cycle. First, the field effect transistor channeled by bilayer graphene (BLG-FET) is exposed to gaseous $\mathrm{O}_{2}$ while the gate voltage $V_{\mathrm{g} \text {,ad }}$ is applied (left panel). Then the system is evacuated and the source-drain conductivity of the BLG-FET is measured by sweeping the gate voltage $V_{\mathrm{g}}$ (right panel). Subsequently gaseous $\mathrm{O}_{2}$ is again introduced, and the cycle is repeated. The gas introduction and evacuation are completed in a shorter time than $\sim 10 \mathrm{~s}$ to prevent additional gas from adsorption. The whole cycle is executed at room temperature. (b) Change of the field effect behavior due to the $\mathrm{O}_{2}$ exposure with $V_{\mathrm{g} \text {,ad }}=0 \mathrm{~V}$ (run 1). The gate voltage giving the minimum conductivity (the charge neutrality point), is shifted from $V_{\mathrm{g}}=V_{\mathrm{CNP}}^{0}(<8 \mathrm{~V})$ (marked by a brown triangle, before $\mathrm{O}_{2}$ exposure) to the positive direction (green triangles) upon $\mathrm{O}_{2}$ exposure. Circles on the curves represent the conductivity at the hole density of $2.5 \times 10^{12} \mathrm{~cm}^{-2}$ that are used to calculate Drude conductivity shown in Figure 4a. (c) The same measurement as in the panel (b) with applying the finite $V_{\mathrm{g} \text {,ad }}$. All the curves are shifted by $-V_{\mathrm{CNP}}^{0}\left(V_{\mathrm{CNP}}^{0}=13,9\right.$, and $11 \mathrm{~V}$ for the run of $V_{\mathrm{g} \text {,ad }}=+80$, +40 , and $-50 \mathrm{~V}$, respectively) in $V_{\mathrm{g}}$ direction, i.e., the charge neutrality points of the pristine graphene without the adsorbed oxygen are taken as zero gate voltage. The top panel of (c) represents the $\sigma$ vs $V_{\mathrm{g}}-V_{\mathrm{CNP}}^{0}$ for the pristine graphene. The changes of $\sigma$ vs $V_{\mathrm{g}}-V_{\mathrm{CNP}}^{0}$ curve after a single and a double exposure to $\mathrm{O}_{2}$ (the time duration of a single exposure is $30 \mathrm{~s}$ ) are shown in the center and the lower panel of (c), respectively. Filled circles indicate $V_{\text {shift }}=V_{\mathrm{CNP}}-V_{\mathrm{CNP}}^{0}$ (the shift of the CNP) for each curve.

$c_{\mathrm{g}}$ is the capacitance per unit area for the back-gated graphene FET on 300 nm-thick $\left.\mathrm{SiO}_{2}\right)^{22}$, It is in the range of the values for BLG-FET in two-probe configuration previously reported ${ }^{6}$, and therefore we confirm that the graphene of the present BLG-FET has few defects that may extremely enhance the chemical reactivity of graphene ${ }^{30}$.

Next we exposed the BLG-FET to 1 atm of highpurity (>99.9995\%) oxygen in the measurement chamber at room temperature. Instead of measuring conductivity with graphene kept in the $\mathrm{O}_{2}$ environment, we performed the short-time interval exposure-evacuation cycles schematically shown in Figure 1a; $\mathrm{O}_{2}$ exposure was done under the dc gate voltage $V_{\mathrm{g} \text {,ad }}$, followed by rapid evacuation in less than $10 \mathrm{~s}$ (the physisorbed $\mathrm{O}_{2}$ molecules would be removed immediately without charge transfer), and eventually the $\sigma$ vs $V_{\mathrm{g}}$ measurement was done with sweeping $V_{\mathrm{g}}$ under vacuum. In this cycle, we can rule out the possibility of the additional oxygen adsorption during sweeping gate voltage for the $\sigma$ vs $V_{\mathrm{g}}$ measurement since the system was evacuated then. In addition, we found that the $\sigma$ vs $V_{\mathrm{g}}$ curve did not vary under vacuum at room temperature at least for more than several hours, so that we can also rule out the possibility of the oxygen desorption during $\sigma$ vs $V_{\mathrm{g}}$ measurement (taking ca. 10 min to obtain a single $\sigma$ vs $V_{\mathrm{g}}$ curve). Therefore, just repeating the cycles substantially realizes the long-time $\mathrm{O}_{2}$ exposure under $V_{\mathrm{g} \text {,ad }}$, the length of which is denoted by total $\mathrm{O}_{2}$ exposure time, $t$. Figure 1b shows the change in $\sigma$ vs $V_{\mathrm{g}}$ by repeating the $\mathrm{O}_{2}$ exposure-evacuation cycles without applying gate voltage during $\mathrm{O}_{2}$ exposure (run $1, V_{\mathrm{g} \text {,ad }}=0 \mathrm{~V}$ ). The shift of the charge neutrality point by the amount of $V_{\text {shift }}(t)=V_{\mathrm{CNP}}(t)-V_{\mathrm{CNP}}^{0}$ toward the positive di- 


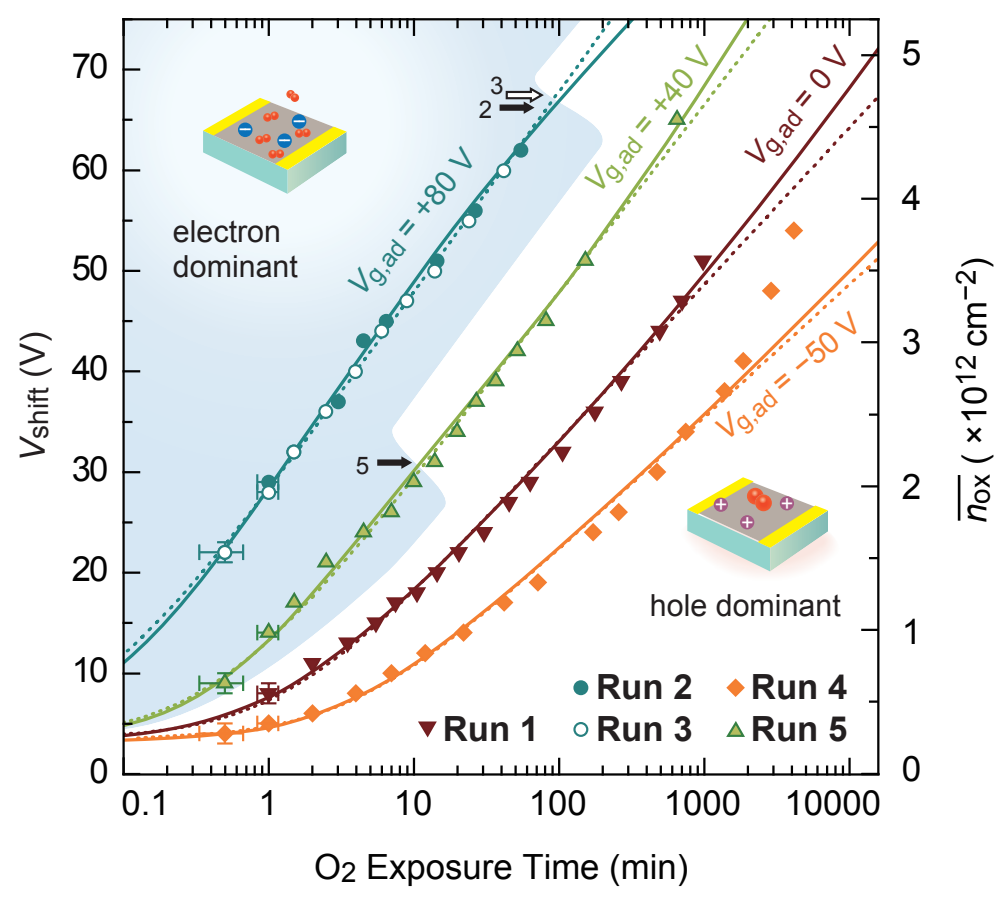

FIG. 2: Time dependence of $V_{\text {shift }}$ and doping density $\overline{n_{\mathrm{ox}}}$ with doping due to the $\mathrm{O}_{2}$ exposure under application of various $V_{\mathrm{g}, \text { ad }}$. Solid and dotted curves are the fits based on the $\mathrm{H}$ kinetics and the $\mathrm{P}$ kinetics (see text), respectively. The curve fitting is made in the range of exposure time below $2000 \mathrm{~min}$. The electron-dominant region, where the Fermi level of BLG is higher than the CNP $\left(V_{\mathrm{g} \text {,ad }}>V_{\mathrm{CNP}}\right)$, is painted blue and the hole-dominant region $\left(V_{\mathrm{g} \text {,ad }}<V_{\mathrm{CNP}}\right)$ is painted white. Carrier type is inverted between electron and hole at the point indicated by arrows (numbers aside correspond to the run number) during the evolution of the oxygen adsorption.

rection was observed (Figure 1b), which represents hole doping to graphene. Prolonged exposure brought further hole doping, and eventually the doping density (induced charge by the oxygen adsorption) $\overline{n_{\mathrm{ox}}}(t)=\left(c_{\mathrm{g}} / e\right) V_{\text {shift }}(t)$ reached more than $5 \times 10^{12} \mathrm{~cm}^{-2}$ within a time scale of $10^{3} \mathrm{~min}$. Note that any hysteresis as observed in graphene FET in moist atmosphere ${ }^{29,31-34}$ was not found in the observed $\sigma$ vs $V_{\mathrm{g}}$ curve, so that we can uniquely determine $V_{\mathrm{CNP}}(t)$ as a function of $t$. Another remarkable feature is the hole conductivity in highly doped regime, $V_{\mathrm{g}}-V_{\mathrm{CNP}}(t)<-40 \mathrm{~V}$ (i.e., $|n|>3 \times 10^{12} \mathrm{~cm}^{-2}$ ). The $\sigma$ vs $V_{\mathrm{g}}$ curve distorted and exhibited the sublinear dependence in this regime for the pristine BLG-FET as can be seen in Figure 1b. Yet it disappeared only after the exposure to $\mathrm{O}_{2}$ for $1 \mathrm{~min}$, whereas the carrier doping has not proceeded much at that time. Thus this rapid change in conductivity feature is discriminated from the slower change causing the shift of the CNP; one possibility is that the former is due to rapid oxidation of the metalgraphene interface ${ }^{35,36}$, which does not shift the Fermi level of graphene but asymmetrically varies the conductivity.

Both $V_{\mathrm{CNP}}$ and the mobility of the BLG-FET with the oxygen adsorbed were reset to the value for the pristine BLG-FET by annealing the $\mathrm{O}_{2}$-exposed BLG-FET in vacuum at $200^{\circ} \mathrm{C}$, indicating that $\mathrm{O}_{2}$ desorption readily proceeds at high temperature without making any defects. By virtue of this reversibility of the oxygen ad- sorption, we can repeat the conductivity measurements in the exposure-evacuation cycles as described above for the same device and compare the results. We additionally carried out four consecutive measurements under the same condition except that a finite gate voltage $V_{\mathrm{g} \text {,ad }}$ was applied during $\mathrm{O}_{2}$ exposure; $V_{\mathrm{g} \text {,ad }}$ was $+80 \mathrm{~V}$ (run 2 and run 3), $-50 \mathrm{~V}$ (run 4) and $+40 \mathrm{~V}$ (run 5). Figure 1c represents the change of the gate-dependent conductivity $\sigma$ vs $V_{\mathrm{g}}-V_{\mathrm{CNP}}^{0}$ at the initial step of the runs 2,4 , 5: before $\mathrm{O}_{2}$ exposure, (i.e., after vacuum annealing) and after the first and second exposure-evacuation cycles (the duration time for $\mathrm{O}_{2}$ exposure in each cycle is 30 s, i.e., $t=0.5,1 \mathrm{~min}$, after the first and second cycle, respectively). All the $\sigma$ vs $V_{\mathrm{g}}-V_{\mathrm{CNP}}^{0}$ curves collapsed onto almost the identical curve in the pristine graphene as shown in the top panel of Figure 1c. Since $V_{\text {CNP }}^{0}$ was within $10 \pm 3 \mathrm{~V}$ for each run (see the caption of Figure 1), the BLG-FET was realized to exhibit its pristine feature before $\mathrm{O}_{2}$ exposure. After the $\mathrm{O}_{2}$ exposure (the center and the bottom panel of Figure 1c) the gate-dependent conductivity changes similarly as was also observed for run 1 (Figure 1b), but the effect of applying $V_{\mathrm{g} \text {,ad }}$ during $\mathrm{O}_{2}$ exposure is marked by the clear difference in $V_{\text {shift }}(t)$. There is a tendency that $V_{\text {shift }}(t)$ is larger for higher $V_{\mathrm{g} \text {,ad }}$ and smaller for lower $V_{\mathrm{g}, \text { ad }}$, indicating that hole doping proceeds more intensively to graphene with higher Fermi level. This trend is pronounced on the increase in the exposure time, as confirmed by comparing the center and 


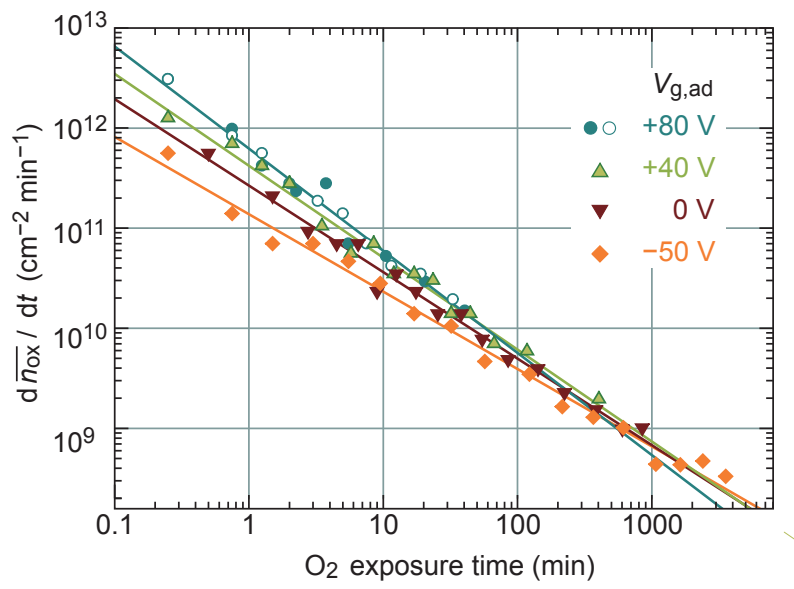

FIG. 3: Double logarithmic plot of time dependence of $\mathrm{d} \overline{n_{\mathrm{ox}}} / \mathrm{d} t$. Symbols are taken in common with Figure 2. We estimate $\mathrm{d} \overline{n_{\mathrm{ox}}} / \mathrm{d} t$ from the differential between the neighboring data points for each run in Figure 2. Lines are linear fits for all the differential data. The slope of each curve gives $u=$ $1.02,0.91,0.86$, and $0.77\left(\mathrm{~d} \overline{n_{\mathrm{ox}}} / \mathrm{d} t \propto t^{-u}\right)$ for the gate voltage $V_{\mathrm{g}, \text { ad }}=+80,+40,0$, and $-50 \mathrm{~V}$, respectively.

the bottom panel of Figure 1(c).

We tracked the temporal evolution of the gatedependent conductivity over a wide time range between $10^{0}-10^{3} \mathrm{~min}$. Figure 2 represents $V_{\text {shift }}$ for runs $1-5$ with respect to $\mathrm{O}_{2}$ exposure time, in which the corresponding doping density owing to the oxygen adsorption, $\overline{n_{\mathrm{ox}}}$, is also shown in the right axis. The tendency that the high $V_{\mathrm{g}, \text { ad }}$ leads to rapid doping can be seen clearly over a whole time range; e.g., to reach the doping level of $V_{\text {shift }}=40 \mathrm{~V}$, it took ca. 300 min for nonbiased BLGFET. In contrast, hole doping is so enhanced for the BLG-FET of $V_{\mathrm{g}, \text { ad }}=80 \mathrm{~V}$ that it took only $4 \mathrm{~min}$, on the other hand so suppressed for that of $V_{\mathrm{g} \text {,ad }}=-50 \mathrm{~V}$ that it took more than $1000 \mathrm{~min}$. The doping density increases almost linearly with respect to $\log t$ for $V_{\mathrm{g}, \text { ad }}=+80 \mathrm{~V}$ and $+40 \mathrm{~V}$, whereas superlinearly for $V_{\mathrm{g}, \mathrm{ad}}=0 \mathrm{~V}$ and $-50 \mathrm{~V}$. The plots for runs 2 and 3 , having common $V_{\mathrm{g}, \text { ad }}=+80 \mathrm{~V}$, are completely on the same line, which verifies that the thermal annealing in vacuum for the reproducing of the undoped state in the BLG does not affect the behavior of adsorption.

Figure 3 shows the time dependence of the doping rate $\mathrm{d} \overline{n_{\mathrm{ox}}} / \mathrm{d} t$ estimated from the differential between the neighboring data points in Figure 2. It is obvious that the doping rate changes in accordance with $\mathrm{d} \overline{n_{\mathrm{ox}}} / \mathrm{d} t \propto t^{-u}$. The power $u$ is dependent on $V_{\mathrm{g}, \mathrm{ad}} ; u \approx 1$ for $V_{\mathrm{g}, \text { ad }}=+80 \mathrm{~V}$ and it decreases for the runs with lower $V_{\mathrm{g} \text {,ad }}$. This deviates from the conventional Langmurian kinetics for molecular adsorption which would give $\mathrm{d} \overline{n_{\mathrm{ox}}} / \mathrm{d} t \propto \exp (-t / \tau)$ with a constant $\tau$.

Careful verification is necessary to inquire the gatevoltage-dependent and non-Langmurian temporal change of the molecular doping since the rate for doping density $\mathrm{d} \overline{n_{\mathrm{ox}}} / \mathrm{d} t$ is related to both of the rate for the chemisorption of molecules ( $\mathrm{d} N_{\mathrm{ox}} / \mathrm{d} t$, where $N_{\text {ox }}$ is the areal density of the adsorbed oxygen molecules) and the transferred charge per adsorbed molecule (the charge/molecular ratio, $Z$ ). Therefore, we analyze the mobility that includes the information of the scattering mechanism of the conducting electrons and the charge of the adsorbed molecules. Within a standard Boltzmann approach ${ }^{37}$, the mobility is changed inversely proportional to the density of the scattering centers (i.e., the adsorbed molecules), $N_{\text {ox }}$. In the realistic case, the inverse mobility is given as a function of $N_{\mathrm{ox}}$ and the carrier density $n$, which reads ${ }^{5}$

$$
\frac{1}{\mu\left(n, N_{\text {ox }}\right)}=\frac{N_{\text {ox }}}{C(n)}+\frac{1}{\mu_{0}(n)}
$$

Here $\mu_{0}(n)$ represents the mobility of the pristine graphene without the adsorbed oxygen. The coefficient $C(n)$ represents the feature of carrier scattering by the adsorbed oxygen. On the one hand, the charged-impurity scattering $^{38}$ gives $C(n) \propto\left[1+6.53 \sqrt{n}\left(d+\lambda_{\mathrm{TF}}\right)\right] / Z^{2}$ for the BLG in the low-carrier-density regime and in the limit of $d \rightarrow 0$ within the Thomas-Fermi approximation $^{38}$, where $d$ is the distance between the impurities and the center of the two layers of the BLG (see the inset of Figure 4a for the definition), and the screening length $\lambda_{\mathrm{TF}}=\kappa \hbar^{2} / 4 m^{*} e \approx 1 \mathrm{~nm}(\kappa$ : dielectric constant $)^{38}$. On the other hand, the short-range deltacorrelated scatterers give the constant $C(n) \equiv C_{\mathrm{s}}{ }^{38}$, or the strong impurities with the potential radius $R$ give $C(n) \propto[\ln (R \sqrt{\pi n})]^{2}$ in the off-resonance condition ${ }^{39}$, which is a decreasing function of $n$ in the regime of $n \sim 10^{12} \mathrm{~cm}^{-2}$ taking $R$ to be several angstroms. As for the relation between the concentration of the adsorbed oxygen molecules $N_{\text {ox }}$ and the $\mathrm{O}_{2}$-induced doping density $\overline{n_{\mathrm{ox}}}$, we assume that the charge $Z e$ of each adsorbed oxygen molecule dopes the carriers $-Z e$ in the BLG, notably, $\overline{n_{\mathrm{ox}}}=-Z N_{\mathrm{ox}}$. To be exact, the amount of the induced charge is not such a simple function ${ }^{6}$ proportional to the number of adsorbed molecules due to the energy-dependent DOS of BLG ${ }^{40,41}$ and the anomalistic screening effect therein ${ }^{5,6,42}$. Yet in the low energy regime of BLG where the DOS is envisaged to be constant, the assumption above is appropriate.

Figure $4 \mathrm{a}$ shows the inverse Drude mobility $\mu^{-1}$ vs $\overline{n_{\mathrm{ox}}}$ plots at the carrier density of $n=2.5 \times 10^{12} \mathrm{~cm}^{-2}$ (marked by the open circles in Figure $1 \mathrm{~b}$ for run 1: $V_{\mathrm{g}, \mathrm{ad}}=$ $0 \mathrm{~V})$ for $V_{\mathrm{g} \text {,ad }}=+80,+40,0$, and $-50 \mathrm{~V}$. Linear increase in $\mu^{-1}$ with respect to $\overline{n_{\mathrm{ox}}}$ was found. This, along with the linearity between $\mu^{-1}$ and $N_{\text {ox }}$ given by Eq. (1), implies that $Z$ is not a function of $N_{\text {ox }}$, i.e., invariant against the increase of the adsorbed molecules. Interestingly, the slope of $\mu^{-1}$ vs $\overline{n_{\mathrm{ox}}}$ plot (the inverse of the slope corresponds to $C(n)|Z|$ in Eq. (1)) depends on $V_{\mathrm{g} \text {,ad }}$. Note that before $\mathrm{O}_{2}$ was introduced $\left(\overline{n_{\mathrm{ox}}}=0\right)$, we observed $\mu^{-1} \approx 28 \mathrm{~V} \mathrm{~s} \mathrm{~m}^{-2}$ irrespective of $V_{\mathrm{g}, \text { ad }}$, and thus the difference in the mobility by $V_{\mathrm{g}, \text { ad }}$ genuinely results from 

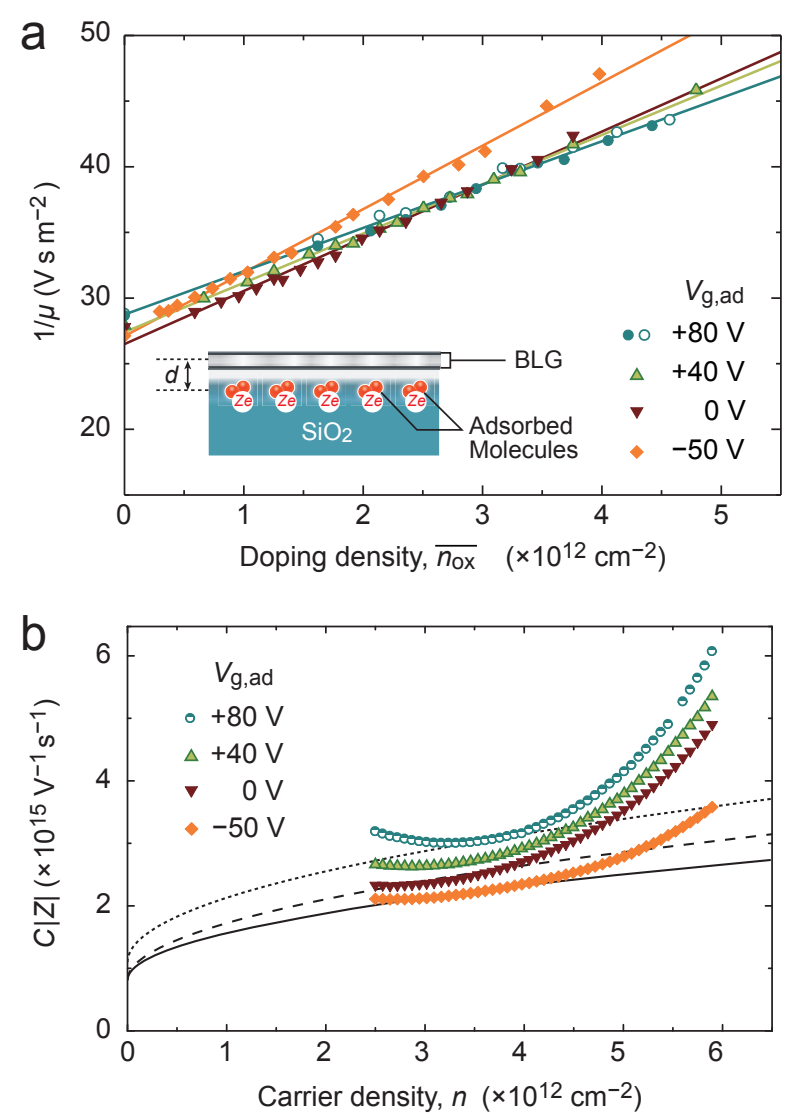

FIG. 4: (a) Inverse mobility $\mu^{-1}$ vs the doping density $\overline{n_{\mathrm{ox}}}$ for each run in Figure 2. Symbols are taken in common with Figure 2. Lines are linear fits (as for $V_{\mathrm{g}, \text { ad }}=+80 \mathrm{~V}$, the data for both run 2 and run 3 are included). Inset: Adsorbed oxygen species with charge $Z e$ positioned at the distance $d$ away from the center of BLG. (b) $C|Z|$ vs the carrier density $n$ (see Eq. (1)). For $V_{\mathrm{g} \text {, ad }}=+80 \mathrm{~V}, C|Z|$ is acquired by gathering the data for run 2 and run 3. Theoretical results based on a charged-impurity scattering mechanism are shown with the various distance $d$ and charge/molecular ratio $Z$ : the solid line represents the result for $d=0.43 \mathrm{~nm}$ and $Z=0.38$, the dashed line for $d=0.73 \mathrm{~nm}$ and $Z=0.38$, and the dotted line for $d=0.43 \mathrm{~nm}$ and $Z=0.28$, respectively.

the adsorbed oxygen instead of other unintentional impurities on the BLG or the $\mathrm{SiO}_{2}$ substrate. In Figure 4b, the inverse of the slope, $C(n)|Z|$, is shown for the various carrier densities, $n$. Therein we omit the data in the low carrier regime of $n<2.5 \times 10^{12} \mathrm{~cm}^{-2}$, in which the residual carriers due to electron-hole puddles cannot be disregarded and the carrier density $n$ (and thus also the Drude mobility) cannot be correctly estimated only by considering the gate electric field effect ${ }^{43}$. Similarly to the charged impurity model rather than otherwise, $C(n)|Z|$ is increasing with $n$. The dependence experimentally observed, however, still deviates from the theoretical calculated results within the charged impurity model plotted in Figure $4 \mathrm{~b}$ for various $d$ and $Z$ (assuming $d$ and $Z$ are invariant to $n$ ).
The difference in $C(n)|Z|$ depending upon $V_{\mathrm{g} \text {,ad }}$ indicates that the electronic polarity of graphene varies the adsorption states of oxygen molecules, leading to the variation of $d$ and $Z$. When the positive (negative) $V_{\mathrm{g}, \text { ad }}$ is applied, negative (positive) carrier is electrically induced on graphene, which may modify the interaction between graphene and the adsorbed oxygen molecules with the negative charge, e.g., the Coulomb interaction and the overlap of the orbitals. Eventually, the stable adsorption state is varied by $V_{\mathrm{g}, \text { ad }}$, leading to the difference in mobility. Besides, let us recall that conductivity measurement process is set apart from the $\mathrm{O}_{2}$ adsorption process and that constant $V_{\mathrm{g} \text {,ad }}$ is not applied when the mobility is measured (Figure 1a). Accordingly, whereas the stable adsorption state of oxygen during the conductivity measurement may differ from that during adsorption, the adsorbed oxygen molecules are kept in the former state during conductivity measurement, and the mobility varying by $V_{\mathrm{g}, \text { ad }}$ is actually observed. This indicates that the energetic barrier exists for charge redistribution between graphene and the adsorbed oxygen molecules (shown below), and once the adsorption is accomplished, the charge $Z e$ on each adsorbed oxygen molecules will not immediately change just after switching on/off the gate voltage. One possible reason for the deviation between the experimental results and theoretical curve is that $d$ varies accompanied with the change in $n$ (or sweeping $V_{\mathrm{g}}$ ). Yet actually, since modifying $d$ by several angstroms results in the small change in $(C(n)|Z|)$ as shown in Figure 4b, it is necessary to investigate more about the behavior of the adsorbed oxygen molecules in the gate electric field in a the future study.

It is controversial what kind of oxygen species does actually cause the hole doping to graphene. Because the electron affinity of $\mathrm{O}_{2}\left(0.44 \mathrm{eV}^{44}\right)$ is much lower than the work function of graphene $\left(4.6 \mathrm{eV}^{45}\right)$, direct charge transfer between them seems unfavorable. Instead, with an analogy of the charge doping of diamond surface ${ }^{46}$, there is a widely accepted ${ }^{28,33,47}$ hypothesis that the hole doping proceeds through an electrochemical reaction ${ }^{46}$ such as: $\mathrm{O}_{2}+2 \mathrm{H}_{2} \mathrm{O}+4 e^{-}=4 \mathrm{OH}^{-}$, by which the charge transfer is favorable due to the lowered free energy change $\Delta G=-0.7 \mathrm{eV}^{28}$ on the condition that the oxygen pressure is $1 \mathrm{~atm}$ and $\mathrm{pH}=7$. This electrochemical reaction needs the aid of water that is mostly eliminated in the experiment by annealing (we observed no hysteresis in the $\sigma$ vs $V_{\mathrm{g}}$ curve, that is, there are few charge traps often attributed to residual moisture on graphene or its substrate). Yet as for graphene deposited on the hydrophilic $\mathrm{SiO}_{2}$ substrate ${ }^{32}$, it is possible that a small amount of residual water molecules (more than the chemical equivalent of $\mathrm{O}_{2}$ ) are trapped on $\mathrm{SiO}_{2}$ surface or voids, which cannot be easily removed by the vacuum annealing at $200{ }^{\circ} \mathrm{C}$ in comparison with those on graphene surface. We suggest that the electrochemical mechanism is plausible also in our case, yet the adsorbed molecule could be other chemical species than $\mathrm{OH}^{-}$, the charge of which may be dependent on $V_{\mathrm{g} \text {,ad }}$. 
a

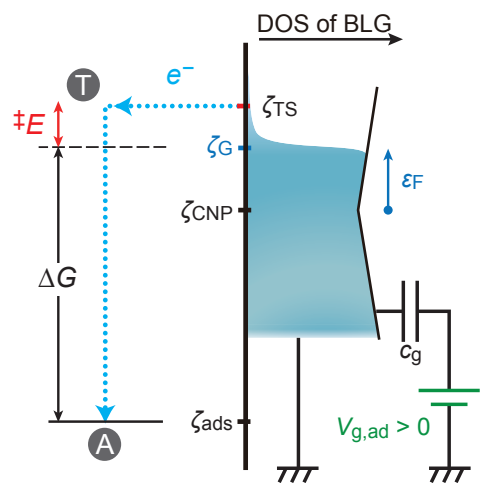

b

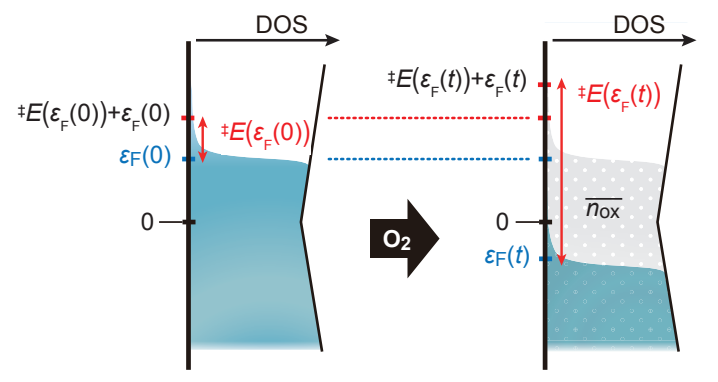

FIG. 5: Schematic energy diagrams of the kinetics of $\mathrm{O}_{2}$ adsorption ( $\mathrm{H}$ kinetics). (a) Path for electron transfer in this model is shown by the blue dotted arrow; electrons in BLG (the electrochemical potential $\zeta_{\mathrm{G}}$ ) are transferred to $\mathrm{O}_{2}$ molecules via the transition state (the circled $\mathrm{T}$ at the level of $\zeta_{\text {TS }}$ ), giving the adsorbed oxygen species (the circled $\mathrm{A}$ at the level of $\left.\zeta_{\text {ads }}\right)$. The activation energy, the free energy change, and the level of the CNP are denoted by ${ }^{\ddagger} E$, $\Delta G$ and $\zeta_{\mathrm{CNP}}$, respectively. The Fermi level is defined by $\varepsilon_{\mathrm{F}}=\zeta_{\mathrm{G}}-\zeta_{\mathrm{CNP}}$. As a demonstration, the case for $V_{\mathrm{g} \text {,ad }}>0$ is presented. (b) Temporal change in the activation energy and the Fermi energy due to the adsorption of oxygen molecules to BLG negatively doped by the positive gate voltage (as in case of panel a). The left panel represents the case before oxygen adsorption $(t=0)$, and the right panel represents the oxygen exposure for the time $t$. Here the energy is measured from the CNP. The red and the blue tick marks denote the level for the transition state and the Fermi level, respectively. Oxygen adsorption lowers the Fermi level, accompanied with the increase in hole doping of $\overline{n_{\mathrm{ox}}}$ (equal to the area of grayed part, $\left.\int_{\varepsilon_{\mathrm{F}}(t)}^{\varepsilon_{\mathrm{F}}(0)} D\left(\varepsilon_{\mathrm{F}}\right) \mathrm{d} \varepsilon_{\mathrm{F}}\right)$, and the activation energy increases according to Eq. (2)

In light of the discussion above, the electrochemical description ${ }^{33,48}$ is expected to be applicable for the observed adsorption kinetics of oxygen to the BLG. Here we premise that the molecular adsorption is determined by the electrochemical potential of graphene, and consider the charge transfer kinetics in an approach based on Butler-Volmer theory ${ }^{49,50}$. The model is schematically depicted in Figure 5. The probability of the adsorption reaction is determined by the electrochemical potential of graphene $\left(\zeta_{\mathrm{G}}\right)$ and that in the equilibrium condition of the oxygen-chemisorption reaction $\left(\zeta_{\text {ads }}\right)$. When
$\Delta G=\zeta_{\mathrm{ads}}-\zeta_{\mathrm{G}}<0$, the electrons favorably transfer from of graphene to the adsorbed oxygen (denoted by "A" in Figure 5a), and the oxygen-adsorption reaction proceeds. For charge transfer, the electrons should go through some energy barrier; we assume that electrons tunnel from BLG to the $\mathrm{O}_{2}$ molecules via a single transition state (denoted by " $T$ "), whose electrochemical potential is $\zeta_{\mathrm{TS}}$. The difference ${ }^{\ddagger} E=\zeta_{\mathrm{TS}}-\zeta_{\mathrm{G}}$ corresponds to the activation energy of the oxygen-chemisorption reaction, which determines the frequency of the electron transfer. Whereas $\zeta_{\mathrm{G}}$ is dependent on the Fermi level $\varepsilon_{\mathrm{F}}$ as $\zeta_{\mathrm{G}}=\varepsilon_{\mathrm{F}}+\zeta_{\mathrm{CNP}}\left(\zeta_{\mathrm{CNP}}\right.$ is the electrochemical potential of the CNP), we envisage that $\zeta_{\mathrm{TS}}$ (or ${ }^{\ddagger} E$ ) is a function of $\varepsilon_{\mathrm{F}}$ as well. In the framework of the Butler-Volmer theory, we obtain the dependence of ${ }^{\ddagger} E$ on $\varepsilon_{\mathrm{F}}$ as

$$
{ }^{\ddagger} E\left(\varepsilon_{\mathrm{F}}+\mathrm{d} \varepsilon_{\mathrm{F}}\right)={ }^{\ddagger} E\left(\varepsilon_{\mathrm{F}}\right)-\alpha \mathrm{d} \varepsilon_{\mathrm{F}},
$$

where $\alpha(>0)$ is a constant related to the "transfer coefficient" in the Butler-Volmer theory that associates the activation energy with the electrochemical potential (not the Fermi energy); thus herein we call $\alpha$ as "pseudo transfer coefficient" (see Supporting Information for detail in the derivation). That is, we have the assumption that the activation energy scales linearly with the Fermi energy. Further assuming that the molecular adsorption rate $\mathrm{d} N_{\text {ox }} / \mathrm{d} t$ is controlled by the electron transfer process and is not strongly affected by other contributions such as molecular diffusion ${ }^{14}$, it is given by

$$
\frac{\mathrm{d} N_{\mathrm{ox}}}{\mathrm{d} t}=\chi D\left(\varepsilon_{\mathrm{F}}+{ }^{\ddagger} E\left(\varepsilon_{\mathrm{F}}\right)\right) f\left(\varepsilon_{\mathrm{F}}+{ }^{\ddagger} E\left(\varepsilon_{\mathrm{F}}\right) ; \varepsilon_{\mathrm{F}}\right),
$$

where $D(\varepsilon)$ is the density of states (DOS) of BLG ( $\varepsilon$ is the energy measured from $\left.\zeta_{\mathrm{CNP}}\right)$ and $f\left(\varepsilon ; \varepsilon_{\mathrm{F}}\right)=[1+$ $\left.\left(\varepsilon-\varepsilon_{\mathrm{F}}\right) / k_{\mathrm{B}} T\right]^{-1}$ is the Fermi-Dirac distribution function. The coefficient $\chi$ does not depend on $\varepsilon_{\mathrm{F}}$ (if the distance between the adsorbing molecules and BLG varied depending on $\varepsilon_{\mathrm{F}}$ or $V_{\mathrm{g} \text {,ad }}$, the tunneling frequency would be affected so that $\chi$ might be dependent on them as well; yet herein we ignore such effect for simplicity). The right-side of Eq. (3) represents the tunneling rate of the electron from the graphene to oxygen at the energy level of transition state, $\varepsilon=\varepsilon_{\mathrm{F}}+{ }^{\ddagger} E\left(\varepsilon_{\mathrm{F}}\right)=\zeta_{\mathrm{TS}}-\zeta_{\mathrm{CNP}}$. According to Eq. (2) and Eq. (3), the molecular adsorption rate is dependent on the Fermi level of graphene. Using them, we can explain both the temporal evolution of the doping rate and its dependence on the gate voltage $V_{\text {g,ad. }}$.

Let us discuss the temporal change in the doping rate. The Fermi level of graphene is lowered with the increase of the adsorbed oxygen molecules, because the positive charge is induced on graphene by the charge $Z e$ they possess. Recalling that $\overline{n_{\mathrm{ox}}}=-Z N_{\mathrm{ox}}$, the doping rate is given by $\mathrm{d} \overline{n_{\mathrm{ox}}} / \mathrm{d} t$. Furthermore, since ${ }^{\ddagger} E \gg k_{\mathrm{B}} T$ is fulfilled as shown later, we also approximate that $f\left(\varepsilon_{\mathrm{F}}+\right.$ $\left.{ }^{\ddagger} E\left(\varepsilon_{\mathrm{F}}\right) ; \varepsilon_{\mathrm{F}}\right) \simeq \exp \left(-{ }^{\ddagger} E\left(\varepsilon_{\mathrm{F}}\right) / k_{\mathrm{B}} T\right)$. Using the relation $\mathrm{d} \overline{n_{\mathrm{ox}}}=-D\left(\varepsilon_{\mathrm{F}}\right) \mathrm{d} \varepsilon_{\mathrm{F}}$, we acquire a formula describing the temporal change of the Fermi level: 


$$
-D\left(\varepsilon_{\mathrm{F}}(t)\right) \frac{\mathrm{d} \varepsilon_{\mathrm{F}}(t)}{\mathrm{d} t}=\frac{p k_{\mathrm{B}} T}{\alpha_{\mathrm{te}}} D\left(\varepsilon_{\mathrm{F}}(t)+{ }^{\ddagger} E\left(\varepsilon_{\mathrm{F}}(t)\right)\right) \exp \left(\alpha_{\mathrm{te}} \frac{\varepsilon_{\mathrm{F}}(t)-\varepsilon_{\mathrm{F}}(0)}{k_{\mathrm{B}} T}\right),
$$

where $\varepsilon_{\mathrm{F}}(t)=\varepsilon_{\mathrm{F}}\left(t, V_{\mathrm{g}, \text { ad }}\right)$, expressing that the Fermi level is a function of the exposure time $t$ and the gate voltage $V_{\mathrm{g}, \text { ad }}$, and $\varepsilon_{\mathrm{F}}(0)=\varepsilon_{\mathrm{F}}\left(0, V_{\mathrm{g}, \mathrm{ad}}\right)$, the Fermi level at $t=0$. We have specifically defined two constants, the pseudo transfer coefficient $\alpha_{\text {te }}$ (the subscript "te" abbreviates "temporal evolution") and

$$
p=-\frac{\alpha_{\mathrm{te}} Z \chi}{k_{\mathrm{B}} T} \exp \left(-\frac{{ }^{\ddagger} E\left(\varepsilon_{\mathrm{F}}(0)\right)}{k_{\mathrm{B}} T}\right)
$$

The right side of Eq. (4) represents the product of the charge transfer frequency and the amount of charge per adsorbed molecule, whereas the left side does the resultant amount of the doped charge. Because BLG (or also single layer graphene) has the low DOS around the CNP compared to metal, the small amount of carrier doping results in the large shift in the Fermi level, which effectively controls the kinetics. Thus the adsorption kinetics is well described by Eq. (4), the equation focusing on the Fermi level. When we envisage that the BLG is approximately described by two-dimensional parabolic dispersion of the free electron, the DOS becomes constant as $D \equiv D_{\mathrm{P}}=\gamma_{\perp} / \pi\left(\hbar v_{\mathrm{F}}\right)^{2}$, where $v_{\mathrm{F}}=(\sqrt{3} / 2) \gamma_{0} a / \hbar$ is the Fermi velocity in SLG, $a=2.46 \AA$ is the in-plane lattice constant, and $\gamma_{0}=3.16 \mathrm{eV}$ and $\gamma_{\perp} \approx 0.4 \mathrm{eV}^{51,52}$ are the intrasheet and intersheet transfer integrals, respectively. In this case (hereafter labeled as P kinetics), Eq. (6) is readily integrated, giving

$$
\overline{n_{\mathrm{ox}}}(t)=-\left\{\varepsilon_{\mathrm{F}}(t)-\varepsilon_{\mathrm{F}}(0)\right\} D_{\mathrm{P}}=\left(\frac{k_{\mathrm{B}} T D_{\mathrm{P}}}{\alpha_{\mathrm{te}}}\right) \ln (1+p t)
$$

Note that Eq. (6) satisfies $\mathrm{d} \overline{n_{\mathrm{ox}}} / \mathrm{d} t \quad \propto$ $\exp \left[-\alpha_{\mathrm{te}} \overline{n_{\mathrm{ox}}}(t) / k_{\mathrm{B}} T D\right]$ and is equivalent to the integrated form of Elovich equation ${ }^{53,54}$, the empirical equation that is widely applicable to chemisorptions onto semiconductors. When the hyperbolic DOS of $\mathrm{BLG}^{41}$ is reflected to Eq. (4), more accurate but more complicated expression of $\varepsilon_{\mathrm{F}}(t)$ is acquired (denoted as $\mathrm{H}$ kinetics), given by

$$
-\frac{\alpha_{\mathrm{te}}}{k_{\mathrm{B}} T} \exp \left(\alpha_{\mathrm{te}} \frac{\varepsilon_{\mathrm{F}}(0)}{k_{\mathrm{B}} T}\right) S\left[\varepsilon_{\mathrm{F}}(0), \varepsilon_{\mathrm{F}}(t)\right]+p t=0,
$$

where $S\left[\varepsilon_{\mathrm{F}}(0), \varepsilon_{\mathrm{F}}(t)\right]$ is a function that depends on the DOS at the Fermi level and that at the transition state level (derived in the Supporting Information).

We performed curve fitting of the experimental results of $\overline{n_{\mathrm{ox}}}(t)$ with Eq. (6) and Eq. (7) for P kinetics and $\mathrm{H}$ kinetics, respectively. The difference in $V_{\mathrm{g} \text {,ad }}$ by runs is simulated by the dependence of $\varepsilon_{\mathrm{F}}(0)$ on $V_{\mathrm{g} \text {,ad }}$ first without considering the gap-opening effect ${ }^{55,56}$, that is, we calculate $\varepsilon_{\mathrm{F}}(0)$ using the relation that the charge of $c_{\mathrm{g}}\left(V_{\mathrm{g}, \text { ad }}-V_{\mathrm{CNP}}^{0}\right)$ doped on BLG by applying $V_{\mathrm{g} \text {,ad }}$ is equal to $\int_{0}^{\varepsilon_{\mathrm{F}}(0)} D(\varepsilon) \mathrm{d} \varepsilon$. Irrespective of the kinetic models, All the theoretical curves are well fitted to the experimental results except those for run 4 in the range above $10^{3}$ min. Eq. (4) is invalid in the first place in the longtime regime in which the adsorption rate is almost as low as the desorption rate, since the present treatment includes no contribution of desorption. The deviation is, however, contrary to the expectation; the desorption should suppress the evolution of the hole doping yet the enhanced doping was actually observed. Thus we suspect that it is due to long-time scale chemisorption of oxygen onto graphene which is ubiquitously observed in carbon materials ${ }^{57,58}$. Note that the several volts of offsets are added in $V_{\text {shift }}$ (corresponding to the doping density of $2 \times$ $10^{11} \mathrm{~cm}^{-2}$ ) to improve the fitting in the time range of $t \leq$ $10^{0} \mathrm{~min}$. This small offset corresponds to a fast reaction that finishes at the very initial stage of adsorption, e.g., due to the reactive chemisorption of $\mathrm{O}_{2}$ to the defect or edge site of graphene ${ }^{59,60}$.

Figure 6 shows the profiles of the fitting results. Practically, the fitting parameters are following two: $\alpha_{\text {te }}$ and $p$, but in order to derive the initial activation energy before the oxygen adsorption, ${ }^{\ddagger} E\left(\varepsilon_{\mathrm{F}}(0)\right)$, from $p$ based on Eq. (6), we briefly assume that the charge/molecular ratio is independent of the gate voltage and determine that $Z \chi \gamma_{\perp}=1 \times 10^{5} \mathrm{eV}^{2} \mathrm{~min}^{-1}$ from the pre-exponential factor in the literature ${ }^{33}, c_{\mathrm{ox}} \nu \kappa_{\mathrm{el}} \sim 10^{17} \mathrm{~cm}^{-2} \mathrm{~s}^{-1}\left(c_{\mathrm{ox}} \nu \kappa_{\mathrm{el}}\right.$ in the literature corresponds to $\chi \gamma_{\perp} / \pi\left(\hbar v_{\mathrm{F}}\right)^{2}$ in this paper). Note that if we assume another value than $Z \chi \gamma_{\perp}=$ $1 \times 10^{5} \mathrm{eV}^{2} \mathrm{~min}^{-1}$, it results in a uniform shift of the calculated ${ }^{\ddagger} E\left(\varepsilon_{\mathrm{F}}(0)\right)$. In addition, the charge/molecular ratio of the adsorbed molecule $Z$ is likely dependent on $V_{\text {g,ad }}$ from the discussion about the mobility, but it leads the shift of ${ }^{\ddagger} E\left(\varepsilon_{\mathrm{F}}(0)\right)$ only by $\sim k_{\mathrm{B}} T=0.026 \mathrm{eV}$. On the one hand, $\alpha_{\mathrm{te}}$ (representing the temporal change of the activation energy) exhibits a significant deviation between the $\mathrm{H}$ kinetics and the $\mathrm{P}$ kinetics(Figure 6a), or depends on the treatment of the DOS of BLG. This deviation is understood as follows: $\mathrm{H}$ kinetics reflects the DOS of BLG that is a monotonically increasing function with respect to $\left|\varepsilon_{\mathrm{F}}\right|$ having a minimum $\left(D=D_{\mathrm{P}}\right)$ at the $\mathrm{CNP}$ (Figure 6(c)), yet $\mathrm{P}$ kinetics does not. Since the downward shift of the Fermi level upon the oxygen adsorption depends on the DOS at the Fermi level, the range of the change in the Fermi level differs between two kinetics models (Figure 6(c)). Thus $\alpha_{\text {te }}$, related with the Fermi level by Eq. (2), is calculated differently. This result is contrasted with the conventional electrochemical reaction on the metal electrodes in which the kinetics is not signif- 

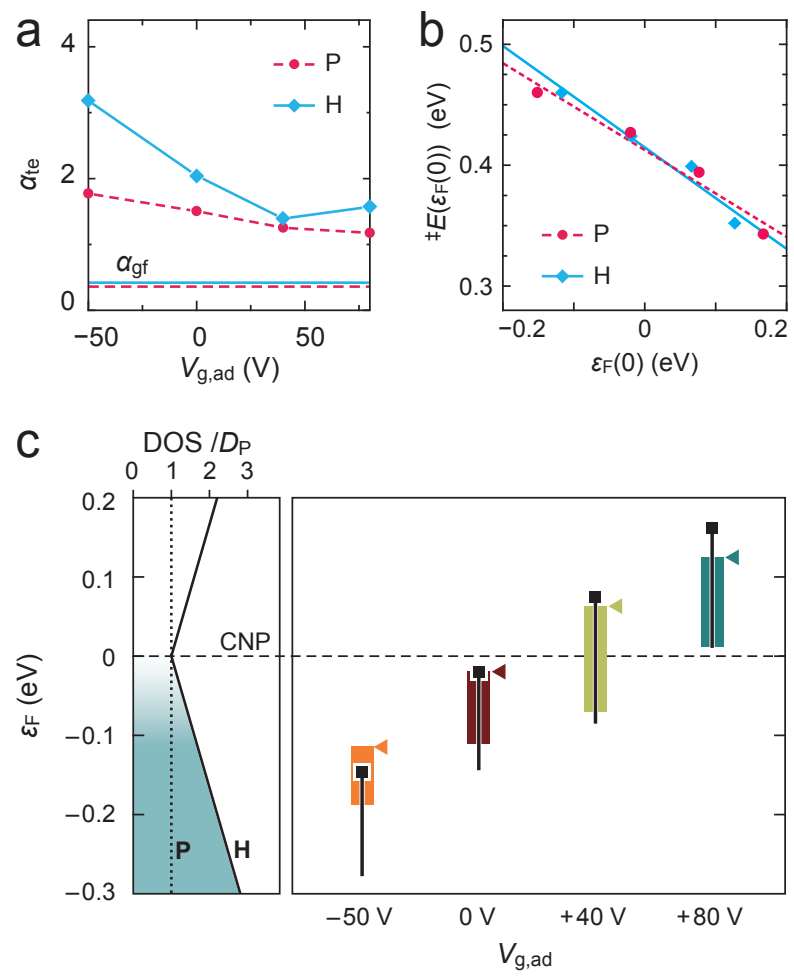

FIG. 6: Summary of parameters obtained by the curve fitting in Figure 2 with $\mathrm{P}$ kinetics $(\mathrm{P})$ and $\mathrm{H}$ kinetics $(\mathrm{H})$ compared. (a) Dependence of the pseudo transfer coefficient $\alpha_{\text {te }}$ on $V_{\mathrm{g}, \text { ad }}$. The pseudo transfer coefficient $\alpha_{\text {gf }}$ acquired by the linear fitting to panel $\mathrm{b}$ is also plotted. (b) Initial activation energy ${ }^{\ddagger} E\left(\varepsilon_{\mathrm{F}}(0)\right)$ plotted as a function of $\varepsilon_{\mathrm{F}}(0)=\varepsilon_{\mathrm{F}}\left(0, V_{\mathrm{g}}\right)$. (c) (Left panel) Density of states (DOS, solid line) for BLG as a function of the Fermi energy, where $D_{\mathrm{P}}$ (DOS at the energy of $\left.\varepsilon_{\mathrm{F}}=0\right)$ is taken as unit of the DOS. The solid line denotes the DOS applied for the $\mathrm{H}$ kinetics. In $\mathrm{P}$ kinetics the DOS is treated as constant; $D=D_{\mathrm{P}}$ (dotted line). (Right panel) Change of the Fermi level by oxygen adsorption as a function of $V_{\mathrm{g} \text {,ad }}$. Bars and boxes represent the range calculated under $\mathrm{P}$ kinetics and $\mathrm{H}$ kinetics, respectively, assuming $\gamma_{\perp}=0.4 \mathrm{eV}$. The Fermi level before the oxygen adsorption $\left(\varepsilon_{\mathrm{F}}(0)\right)$ is at the point indicated by triangles (black squares) for $\mathrm{H}(\mathrm{P})$ kinetics, whereas the Fermi level shifts downward with the increase of the adsorbed oxygen, and eventually reaches the bottom end of the boxes (bars) in the end of the oxygen adsorption for the $\mathrm{H}(\mathrm{P})$ kinetics. The energy is measured from the CNP (dashed line), and the gap-opening effect is not considered.

icantly affected by the DOS of the electrodes and is just owing to the low DOS of the BLG with comparison to the metal. On the other hand, we found that ${ }^{\ddagger} E\left(\varepsilon_{\mathrm{F}}(0)\right)$ is a decreasing function of $\varepsilon_{\mathrm{F}}(0)=\varepsilon_{\mathrm{F}}\left(0, V_{\mathrm{g} \text {,ad }}\right)$ (shown in Figure $6 \mathrm{~b}$, therein we plot $\ddagger E\left(\varepsilon_{\mathrm{F}}(0)\right)$ with respect to $\varepsilon_{\mathrm{F}}(0)$ instead of $\left.V_{\mathrm{g}, \text { ad }}\right)$. From Eq. (2), the slope of the plots in Figure $6 \mathrm{~b}$ corresponds to the pseudo transfer coefficient $\alpha$, and we found that $\alpha_{\mathrm{gf}}=0.36$ and 0.42 for P kinetics and $\mathrm{H}$ kinetics, respectively (the subscript "gf" abbreviates "gate electric field"). Herein we distinguish $\alpha_{\mathrm{gf}}$ from $\alpha_{\text {te }}$; being different from $\alpha_{\text {te }}$ that is calculated based on the temporal Fermi level shift by oxygen adsorption (thus $\alpha_{\text {te }}$ inevitably includes the oxygen adsorption effect), $\alpha_{\mathrm{gf}}$ is acquired by tuning the Fermi level electrically at $t=0$ before oxygen adsorption. We found that $\alpha_{\mathrm{gf}}$ is much smaller than $\alpha_{\mathrm{te}}(\geq 1)$, or rather near 0.5 , a typical transfer coefficient for simple redox reactions ${ }^{48,49}$ (Figure 6a). Specifically, the oxygen adsorption effect included only in $\alpha_{\text {te }}$ but not in $\alpha_{\mathrm{gf}}$ is attributed, e.g., to the electric dipole layer ${ }^{61}$ formed between graphene and the adsorbed molecules and the Coulomb interaction between the adsorbed oxygen molecules. We expect that these effects also raise the activation energy roughly in proportional to the number of molecules $N_{\text {ox }}$ (or the doping density $\overline{n_{\mathrm{ox}}}$ ), and thus we have the expression for the additional adsorption effect: $\mathrm{d}^{\ddagger} E=\xi_{\text {mol }} \mathrm{d} \overline{n_{\text {ox }}}=-\xi_{\text {mol }} D\left(\varepsilon_{\mathrm{F}}\right) \mathrm{d} \varepsilon_{\mathrm{F}}$ ( $\xi_{\text {mol }}$ : the proportional coefficient; herein we use the relation $\mathrm{d} \overline{n_{\mathrm{ox}}}=-D\left(\varepsilon_{\mathrm{F}}\right) d \varepsilon_{\mathrm{F}}$ again). Then we acquire $\alpha_{\mathrm{te}} \simeq \alpha_{\mathrm{gf}}+\xi_{\mathrm{mol}}\left\langle D\left(\varepsilon_{\mathrm{F}}\right)\right\rangle\left(\left\langle D\left(\varepsilon_{\mathrm{F}}\right)\right\rangle\right.$ denotes the average DOS in the range of the Fermi level for each run), which indicates that $\alpha_{\mathrm{te}}$ is large when the DOS is large in the level far away from the CNP (see Figure 6c). Indeed, as shown in Figure $6 \mathrm{a}, \alpha_{\mathrm{te}}$ for $\mathrm{H}$ kinetics shows the $\mathrm{V}$-shaped dependence on $V_{\mathrm{g} \text {,ad }}$ with the minimum for $V_{\mathrm{g}, \text { ad }}=+40 \mathrm{~V}$, which gives the smallest average DOS. Though the kinetics for the molecular adsorption is affected by various effects as mentioned above, we represent them by the parameter $\alpha_{\text {te }}$ and succeed in accounting for the observed kinetics in a facile way.

Finally let us account for the power-law dependence of $\mathrm{d} \overline{n_{\mathrm{ox}}} / \mathrm{d} t \propto t^{-u}$ shown in Figure 3. It is helpful to look on the simpler $\mathrm{P}$ kinetics for the assessment of $\mathrm{d} \overline{n_{\text {ox }}} / \mathrm{d} t$; from Eq. (6), we obtain $\mathrm{d} \overline{n_{\text {ox }}} / \mathrm{d} t \propto p /(1+p t)$. Approximately we have $\mathrm{d} \overline{n_{\mathrm{ox}}} / \mathrm{d} t \propto\left(u\langle t\rangle^{u-1}\right) t^{-u}$ where $u \simeq[1+(1 / p\langle t\rangle)]^{-1} \leq 1$ (the time $\langle t\rangle$ is the center of the expansion of $\ln \left(\mathrm{d} \bar{n}_{\mathrm{ox}} / \mathrm{d} t\right)$ in terms of $\ln t$, and it is a good approximation if $p\langle t\rangle \gg 1$, or otherwise if $p\langle t\rangle \simeq 1$ in the time range such that $\left.t=\left[10^{-1}\langle t\rangle, 10\langle t\rangle\right]\right)$. Since $p$ is intensively dependent on $V_{\mathrm{g} \text {,ad }}$ (recall that (i) $p$ is exponentially decaying against ${ }^{\ddagger} E\left(\varepsilon_{\mathrm{F}}(0)\right)$ as represented in Eq. (5), (ii) ${ }^{\ddagger} E\left(\varepsilon_{\mathrm{F}}(0)\right)$ linearly decreases with respect to $\varepsilon_{\mathrm{F}}(0)$ with the slope of $-\alpha_{\mathrm{gf}}$ as shown in Figure $6 \mathrm{c}$, (iii) $\varepsilon_{\mathrm{F}}(0)$ is an increasing function of $V_{\mathrm{g} \text {,ad }}$. We acquired $p=19.6,2.9,0.98$ and 0.32 for $V_{\mathrm{g}, \text { ad }}=+80,+40,0$, and $-50 \mathrm{~V}$, respectively, by fitting within the $\mathrm{P}$ kinetics model), we can find that $u$ is almost unity for a positively high $V_{\mathrm{g}, \text { ad }}$ and tends be smaller for negatively high $V_{\mathrm{g} \text {,ad }}$ within the time range experimentally scoped, which is consistent with the observed behavior. When the electrochemical mechanism governs the kinetics of the oxygen adsorption, the activation energy of the charge transfer continually increases with the $\mathrm{O}_{2}$ exposure time increasing. This effect leads to non-Langmurian kinetics of the oxygen adsorption and the power-law decrease of $\mathrm{d} \overline{n_{\text {ox }}} / \mathrm{d} t$, even though neither the desorption process nor the saturation limit of adsorption are taken into consideration.

In BLG, it is known that a band gap opens due to the energy difference between two layers ${ }^{9}$. The gate electric 
field as well as the adsorbed oxygen may produce such a strong energy difference that the eventual band gap should affect the time evolution of molecular adsorption. The band gap opening effect is expected to exhibit most prominently when the Fermi level goes across the CNP (at the point shown by arrows in Figure 2), while we cannot find such behavior obviously. We guess it is partly because most of the adsorbed oxygen molecules exist in the interface between graphene and the gate dielectric $\mathrm{SiO}_{2}$. For the band gap opening effect to appear, it is necessary that the gate electric field and the molecular field enhance each other when the Fermi level is near the CNP (i.e., the charge induced by the gate electric field and that by the adsorbed molecules including unintentional residual impurities on the $\mathrm{SiO}_{2}$ substrate are balanced), yet it is possible only when the molecules mainly adsorb on the top surface of BLG, and not for the molecules adsorbed in the interface (Figure S2, Supporting Information). Or it may be partly because the disordered potential due to the impurities in the substrate fluctuates the energy level around which the band gap exists ${ }^{62}$, eventually blurring band gap opening effects and chemical reactivity ${ }^{63}$ of BLG. Details about the band-gap opening effects are discussed in the Supporting Information.

In summary, we investigated the weak chemisorption of $\mathrm{O}_{2}$ molecules on bilayer graphene by measuring its transport properties. The hole doping due to $\mathrm{O}_{2}$ chemisorption is remarkably dependent on the gate voltage, and the amount of the doped carrier increases with $\mathrm{O}_{2}$ exposure time, the rate of which is in accordance with $\propto t^{-u}(u \leq 1)$ rather than with conventional Langmuirian kinetics. We conclude from these that an electrochem- ical reaction governs the $\mathrm{O}_{2}$ chemisorption process, in which the rate of the chemisorption is determined by the Fermi level of graphene, and indeed succeed in accounting for the observed kinetics by the analysis based on the Butler-Volmer theory. We also found that the chemisorbed molecules decrease the mobility of graphene, and interestingly, the mobility change is dependent on the gate voltage applied during the adsorption, indicating that the adsorption state, e.g., transferred charge or distance between a molecule and graphene, can be modified electrically. Graphene, offering a continuously tunable platform for study of chemisorptions on it, realizes the electrical control of the adsorption by gate electric field, a novel and versatile method in which we would explore extensively a wider variety of host-guest interactions between graphene and foreign molecules.

Supporting Information. Additional descriptions about (i) the experimental method, (ii) the electrochemistry-based kinetics model and the mathematical derivation for it, and (iii) an expanded discussion about the gap-opening effects.

\section{Acknowledgments}

The authors acknowledge support from Grant-in-Aid for Scientific Research No. 20001006 from the Ministry of Education, Culture, Sports, Science and Technology, Japan. The authors thank M. Kiguchi, T. Kawakami, K. Yokota, and Y. Kudo for useful discussion.
* Electronic address: mail to: sato.y.an@m.titech.ac.jp

1 R. Balog, B. Jørgensen, L. Nilsson, M. Andersen, E. Rienks, M. Bianchi, M. Fanetti, E. Laegsgaard, A. Baraldi, S. Lizzit, Z. Sljivancanin, F. Besenbacher, B. Hammer, T. G. Pedersen, P. Hofmann, and L. Hornekær, Nat. Mater. 9, 315 (2010).

2 Y. Zhang, A. Kolmakov, S. Chretien, H. Metiu, and M. Moskovits, Nano Lett. 4, 403 (2004).

3 A. H. Castro Neto, F. Guinea, N. M. R. Peres, K. S. Novoselov, and A. K. Geim, Rev. Mod. Phys. 81, 109 (2009).

${ }^{4}$ F. Schedin, A. K. Geim, S. V. Morozov, E. W. Hill, P. Blake, M. I. Katsnelson, and K. S. Novoselov, Nat. Mater. 6, 652 (2007).

5 J. H. Chen, C. Jang, S. Adam, M. S. Fuhrer, E. D. Williams, and M. Ishigami, Nat. Phys. 4, 377 (2008).

6 S. Xiao, J.-H. Chen, S. Adam, E. D. Williams, and M. S. Fuhrer, Phys. Rev. B 82, 041406 (2010).

7 J. H. Chen, C. Jang, M. Ishigami, S. Xiao, W. G. Cullen, E. D. Williams, and M. S. Fuhrer, Sol. Stat. Commun. 149, 1080 (2009).

8 D. W. Boukhvalov and M. I. Katsnelson, Appl. Phys. Lett. 95, 023109 (2009).

9 E. V. Castro, K. S. Novoselov, S. V. Morozov,
N. M. R. Peres, J. M. B. Lopes dos Santos, J. Nilsson, F. Guinea, A. K. Geim, and A. H. Castro Neto, J. Phys.: Condens. Matter 22, 175503 (2010).

10 D. A. Abanin, A. V. Shytov, and L. S. Levitov, Phys. Rev. Lett. 105, 086802 (2010).

11 R. M. Ribeiro, N. M. R. Peres, J. Coutinho, and P. R. Briddon, Phys. Rev. B 78, 075442 (2008).

12 T. Lohmann, K. von Klitzing, and J. H. Smet, Nano Lett. 9, 1973 (2009).

13 V. V. Cheianov, O. Syljuåsen, B. L. Altshuler, and V. I. Fal'ko, EPL 89, 56003 (2010).

14 H. E. Romero, P. Joshi, A. K. Gupta, H. R. Gutierrez, M. W. Cole, S. A. Tadigadapa, and P. C. Eklund, Nanotechnology 20, 245501 (2009).

15 T. O. Wehling, M. I. Katsnelson, and A. I. Lichtenstein, Chem. Phys. Lett. 476, 125 (2009).

${ }^{16}$ K. Takai, S. Eto, M. Inaguma, T. Enoki, H. Ogata, M. Tokita, and J. Watanabe, Phys. Rev. Lett. 98, 017203 (2007).

17 K. M. McCreary, K. Pi, A. G. Swartz, W. Han, W. Bao, C. N. Lau, F. Guinea, M. I. Katsnelson, and R. K. Kawakami, Phys. Rev. B 81, 115453 (2010).

18 K. S. Novoselov, A. K. Geim, S. V. Morozov, D. Jiang, M. I. Katsnelson, I. V. Grigorieva, S. V. Dubonos, and 
A. A. Firsov, Nature 438, 197 (2005).

19 J. Zhou, Q. Wang, Q. Sun, P. Jena, and X. S. Chen, Proc. Natl. Acad. Sci. USA 107, 2801 (2010).

20 P. Saalfrank, J. Chem. Phys. 113, 3780 (2000).

${ }^{21}$ W. Liu, Y. H. Zhao, J. Nguyen, Y. Li, Q. Jiang, and E. J. Lavernia, Carbon 47, 3452 (2009).

22 Y.-H. Lu, L. Shi, C. Zhang, and Y.-P. Feng, Phys. Rev. B 80, 233410 (2009).

23 V. W. Brar, R. Decker, H.-M. Solowan, Y. Wang, L. Maserati, K. T. Chan, H. Lee, c. O. Girit, A. Zettl, S. G. Louie, M. L. Cohen, and M. F. Crommie, Nat. Phys. 7, 43 (2011).

24 J.-H. Parq, J. Yu, Y.-K. Kwon, and G. Kim, Phys. Rev. B 82, 193406 (2010).

${ }^{25} \mathrm{X}$. Tian, J. $\mathrm{Xu}$, and $\mathrm{X}$. Wang, J. Phys. Chem. B 114, 11377 (2010).

26 A. M. Suarez, L. R. Radovic, E. Bar-Ziv, and J. O. Sofo, Phys. Rev. Lett. 106, 146802 (2011).

27 A. A. Kaverzin, S. M. Strawbridge, A. S. Price, F. Withers, A. K. Savchenko, and D. W. Horsell, Carbon 49, 3829 (2011).

${ }^{28}$ H. Pinto, R. Jones, J. P. Goss, and P. R. Briddon, Phys. Status. Solidi. A 207, 2131 (2010).

${ }^{29}$ Y. Yang and R. Murali, Appl. Phys. Lett. 98, 093116 (2011).

${ }^{30} \mathrm{P}$. L. de Andres and J. A. Vergés, Appl. Phys. Lett. 93, 171915 (2008).

${ }^{31}$ Y. Shi, W. Fang, K. Zhang, W. Zhang, and L.-J. Li, Small 5, 2005 (2009).

${ }^{32}$ C. M. Aguirre, P. L. Levesque, M. Paillet, F. Lapointe, B. C. St-Antoine, P. Desjardins, and R. Martel, Adv. Mater. 21, 3087 (2009).

33 P. L. Levesque, S. S. Sabri, C. M. Aguirre, J. Guillemette, M. Siaj, P. Desjardins, T. Szkopek, and R. Martel, Nano Lett. 11, 132 (2010).

34 S. S. Sabri, P. L. Lévesque, C. M. Aguirre, J. Guillemette, R. Martel, and T. Szkopek, Appl. Phys. Lett. 95, 242104 (2009).

35 R. Nouchi and K. Tanigaki, Appl. Phys. Lett. 96, 253503 (2010).

36 T. Yamada, Phys. Rev. B 69, 125408 (2004).

37 S. Das Sarma, S. Adam, E. H. Hwang, and E. Rossi, Rev. Mod. Phys. 83, 407 (2011).

38 S. Adam and S. Das Sarma, Phys. Rev. B 77, 115436 (2008).

39 M. Monteverde, C. Ojeda-Aristizabal, R. Weil, K. Bennaceur, M. Ferrier, S. Guéron, C. Glattli, H. Bouchiat, J. N. Fuchs, and D. L. Maslov, Phys. Rev. Lett. 104, 126801 (2010), Supplementary Material II.

40 E. McCann and V. I. Fal'ko, Phys. Rev. Lett. 96, 086805 (2006).

41 S. Adam and M. D. Stiles,
Phys. Rev. B 82, 075423 (2010).

42 S. Adam, E. H. Hwang, V. M. Galitski, and S. Das Sarma, Proc. Natl. Acad. Sci. USA 104, 18392 (2007).

${ }^{43}$ Y. W. Tan, Y. Zhang, K. Bolotin, Y. Zhao, S. Adam, E. H. Hwang, S. Das Sarma, H. L. Stormer, and P. Kim, Phys. Rev. Lett. 99, 246803 (2007).

44 K. M. Ervin, I. Anusiewicz, P. Skurski, J. Simons, and W. C. Lineberger, J. Phys. Chem. A 107, 8521 (2003).

45 S. J. Sque, R. Jones, and P. R. Briddon, Phys. Status Solidi A 204, 3078 (2007).

46 V. Chakrapani, J. C. Angus, A. B. Anderson, S. D. Wolter, B. R. Stoner, and G. U. Sumanasekera, Science 318, 1424 (2007).

47 S. Ryu, L. Liu, S. Berciaud, Y.-J. Yu, H. Liu, P. Kim, G. W. Flynn, and L. E. Brus, Nano Lett. 10, 4944 (2010).

48 I. Heller, J. Kong, K. A. Williams, C. Dekker, and S. G. Lemay, J. Am. Chem. Soc. 128, 7353 (2006).

49 J. O. M. Bockris and Z. Nagy, J. Chem. Educ. 50, 839 (1973).

50 J. O. Bockris, A. K. N. Reddy, and M. Gamboa-Aldeco, Fundamentals of Electrodics, 2nd ed., Modern Electrochemistry, Vol. 2A (Plenum Press, 2000).

51 W. W. Toy, M. S. Dresselhaus, and G. Dresselhaus, Phys. Rev. B 15, 4077 (1977).

52 M. S. Dresselhaus and G. Dresselhaus, Adv. Phys. 51, 1 (2002).

53 C. Aharoni and F. C. Tompkins, Kinetics of adsorption and desorption and the Elovich equation, edited by D. D. Eley, H. Pines, and P. B. Weisz, Vol. 21 (Academic Press, New York and London, 1970) pp. 1-49.

${ }^{54}$ I. S. Mclintock, Nature 216, 1204 (1967).

${ }^{55}$ T. Ohta, A. Bostwick, T. Seyller, K. Horn, and E. Rotenberg, Science 313, 951 (2006).

56 E. V. Castro, K. S. Novoselov, S. V. Morozov, N. M. R. Peres, J. M. B. L. dos Santos, J. Nilsson, F. Guinea, A. K. Geim, and A. H. Castro Neto, Phys. Rev. Lett. 99, 216802 (2007).

57 N. Kobayashi, T. Enoki, C. Ishii, K. Kaneko, and M. Endo, J. Chem. Phys. 109, 1983 (1998).

${ }^{58}$ G. U. Sumanasekera, G. Chen, K. Takai, J. Joly, N. Kobayashi, T. Enoki, and P. C. Eklund, J. Phys. Condens. Matter 22, 334208 (2010).

59 X. Wang and H. Dai, Nat. Chem. 2, 661 (2010).

60 F. M. Koehler, A. Jacobsen, K. Ensslin, C. Stampfer, and W. J. Stark, Small 6, 1125 (2010).

61 G. Giovannetti, P. A. Khomyakov, G. Brocks, V. M. Karpan, J. van den Brink, and P. J. Kelly, Phys. Rev. Lett. 101, 026803 (2008).

${ }^{62}$ G. M. Rutter, S. Jung, N. N. Klimov, D. B. Newell, N. B. Zhitenev, and J. A. Stroscio, Nat. Phys. 7, 649 (2011).

63 R. Sharma, J. H. Baik, C. J. Perera, and M. S. Strano, Nano Lett. 10, 398 (2010). 